\title{
Resistor Loaded EBG Surfaces for Slot Antenna Design*
}

\author{
Shenyi Cao, Yunqi Fu \\ College of Electronic Science and Engineering, National University of Defense Technology, Changsha, China. \\ Email: shenyicao@gmail.com, yunqifu@nudt.edu.cn \\ Received May $21^{\text {st }}, 2013$; revised June $22^{\text {nd }}, 2013$; accepted July $2^{\text {nd }}, 2013$ \\ Copyright (C) 2013 Shenyi Cao, Yunqi Fu. This is an open access article distributed under the Creative Commons Attribution License, \\ which permits unrestricted use, distribution, and reproduction in any medium, provided the original work is properly cited.
}

\begin{abstract}
We present a resistor loaded EBG surface (REBG) for antenna design. Specifically, lumped resistors are embedded between periodic metal patches of mushroom like EBG structures. Surface wave propagation along the REBG surface is analyzed as a periodically loaded transmission line model and low transmission bandgap is identified and experimentally verified. The reflection properties of REBG surface to the incident electromagnetic waves are also studied. Slot antenna is placed within the REBG surface. The REBG surface is used to suppress surface waves across the antenna aperture, resulting in radiation performance improvement. Concurrently, the REBG surface can also absorb electromagnetic energy to reduce antenna radar cross section (RCS).
\end{abstract}

Keywords: EBG; Antenna; RCS

\section{Introduction}

Electromagnetic bandgap (EBG) structures have been extensively explored in recent years. The most popular of them is the mushroom like EBG structure printed on a microstrip substrate composed of metal islands and vertical connections to the ground plane [1]. This type of EBG structure exhibits bandgap to suppress surface wave propagation and in-phase reflection coefficient. It has been shown $[2,3]$ that suppression of surface waves leads to improved radiation efficiency and antenna gain. Further, side lobes can be reduced by eliminating surface wave scattering from edges. Mutual coupling in phased arrays can also be reduced using EBG structures [4] and scan blindness can be suppressed [5,6].

EBG structures have also been used for antenna radar cross section (RCS) control. For example, a metallic EBG based radome was proposed in [7] to reduce antenna RCS, but worked only when the antenna was switched off. Their in-phase reflection properties have also been exploited to realize ultra-thin radar absorbing materials (RAM). Specifically, the EBG surface can be covered by a lossy dielectric layer [8] or loaded with lumped resistors placed between the adjacent metal patches [9]. In [10] a similar RAM structure has been used for antenna RCS reduction by welding resistors interconnecting the metal patches. However, this design

\footnotetext{
*This work was supported by the Science Foundation for New Century
} Excellent Talents in University of China (Grant No. NCET-10-0894). did not suppress surface waves at the RCS reduction frequency because it did not include vias to the substrate.

In this paper, we present a slot antenna design based on resistor loaded EBG (REBG) surfaces. Waveguide slot arrays in presence of REBG surfaces have already been presented in [11]. This REBG surface did exhibit simultaneously surface wave suppression and absorption of the incident wave over a specific frequency band. Therefore, side lobes could be reduced by suppressing surface wave radiation. The RCS was concurrently reduced.

We also considered the analysis of REBG surfaces. Specifically, the surface wave propagation characteristics of REBG are analyzed using the periodically loaded transmission line model. An optimization of the REBG based slot antenna is considered in terms of its return loss, side lobes and antenna RCS. Finally, a comparison of antennas on EBG and REBG ground planes is presented.

\section{Resistor Loaded Mushroom EBG Structure}

\subsection{Structure and Equivalent Circuit Model}

The geometry of the REBG structure is displayed in Figure 1. Square patches of width $w$ are periodically printed on a grounded substrate (having thickness $h$ and relative permittivity $\varepsilon_{r}$ ). These patches are separated by a gap $g$ and lumped resistors of resistance $R$ are welded between adjacent patches. The circuit model of the REBG surface could then be represented by a parallel RLC circuit as in 


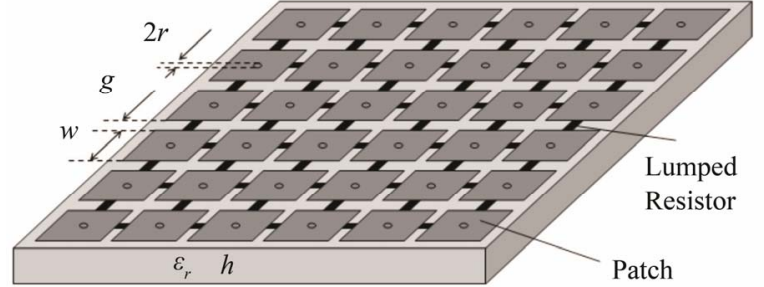

Figure 1. EBG structure loaded with lumped resistors embedded between adjacent square metal patches.

Figure 2. Thus, the associated surface impedance is

$$
Z_{s}=1 /(1 / R+j \omega C+1 / j \omega L)
$$

where $L$ and $C$ are the same as those of an unloaded EBG structure [1] having the geometrical parameters, $w=6 \mathrm{~mm}, g=1 \mathrm{~mm}, r=0.4 \mathrm{~mm}, \varepsilon_{r}=5.8, h=2.5 \mathrm{~m}$, $R=377 \Omega$. Based on these values, a plot of the impedance vs. frequency is given in Figure 2. As seen, the resonance frequency of the unloaded EBG structure is $5.89 \mathrm{GHz}$ (using the circuit model). Of importance is that the impedance characteristics of the REBG surface are quite different than those of the unloaded EBG structure. Specifically, the reactance is zero at the resonance frequencies for the parallel RLC circuit and does not act as a high impedance surface any more. But, the surface impedance is inductive below the resonance frequency and capacitive above it, i.e. the same as the unloaded EBG surface. Therefore, we can still expect a surface wave bandgap. Nevertheless, the lower impedance makes the bandgap much weaker near resonance. Further, the non-zero resistance absorbs surface waves. But as resistance decreases away from resonance, absorption decreases too. Considering these two aspects, the REBG surface can still show a low transmission frequency bandgap.

\subsection{Surface Wave Characteristics of REBG Surface}

Surface wave propagation was examined using the periodically loaded transmission lines, the same procedure as in [12] (see Figure 3). Specifically, the REBG surface was modelled as a transmission line loaded with a lumped impedance $Z$, composed of $Z_{p}$ (input impedance of the unit transmission line) in parallel with $C$ (coupling capacitor between the neighboring patches) and $R$. The chosen period was necessarily $a=w+g$, implying a propagation constant $\gamma$ satisfying the transcendental equation

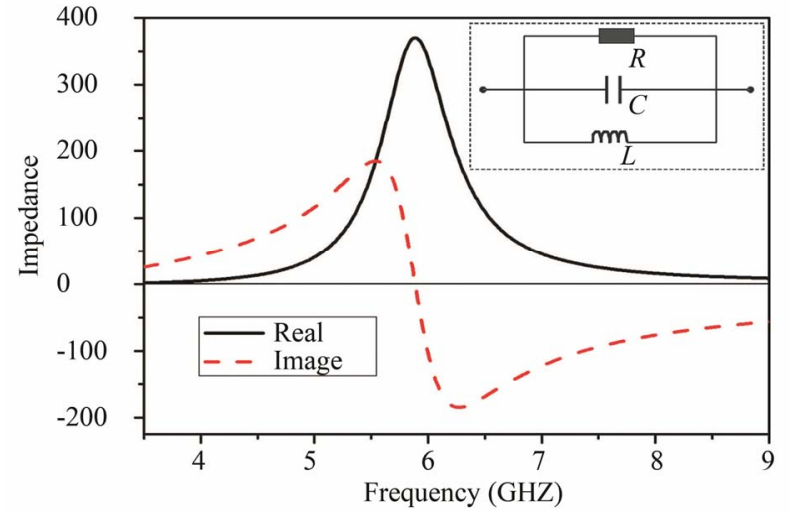

Figure 2. Surface impedance of the REBG structure calculated using the parallel RLC circuit.

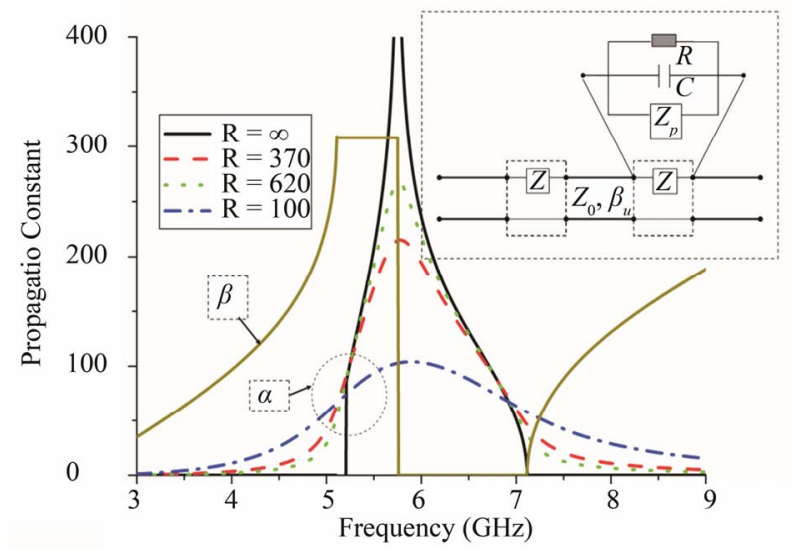

Figure 3. Propagation constants of the REBG surface calculated using the transmission line circuit model.

$$
\cosh (\gamma a)=\cos \left(\beta_{u} a\right)+j Z \sin \left(\beta_{u} a\right) / 2 Z_{0}
$$

In Equation (2), $Z_{0}$ and $\beta_{u}$ refer to the characteristic impedance and phase constant of the unloaded structure (referring to a microstrip line of different width/thickness ratios). Substituting for $\gamma=\alpha+j \beta$, Equation (2) becomes

For unloaded EBG surfaces, $\beta$ is obtained by solving a single equation after setting $\alpha=0$. But for the REBG surface, $\alpha$ or $\beta$ are both non-zero. The unloaded EBG structures were analyzed by setting $R=\infty$. Solving the pair (3), it can be seen from Figure 3 for some frequencies $\beta$ is zero or $n \pi / a$. This corresponds to the bandgap of the unloaded EBG structure.

The REBG attenuation constants for $R=100 \Omega, 370 \Omega$ and $620 \Omega$ are also given in Figure 3. Clearly, $\alpha$ is reduced for smaller $R$. Of interest is that for $R>370 \Omega$, the attenuation constant almost reaches the unloaded $(R=\infty)$

$$
\left\{\begin{array}{l}
\cosh (\alpha a) \cos (\beta a)=\cos \left(\beta_{u} a\right)-\operatorname{Im}(Z) \sin \left(\beta_{u} a\right) / 2 Z_{0} \\
\sinh (\alpha a) \sin (\beta a)=\operatorname{Re}(Z) \sin \left(\beta_{u} a\right) / 2 Z_{0}
\end{array}\right.
$$


EBG values except near the peak around the resonance frequency. It can also be seen that the bandwidth of the REBG surface is larger than that of the unloaded one (as the resistor lowers the $\mathrm{Q}$ factor).

Three REBG samples were fabricated and measured. Two of them were REBG structures with resistor $R=370$ $\Omega$ and $620 \Omega$ and the other was an unloaded EBG structure $(R=\infty)$. The surface wave transmission coefficients across the EBG and REBG surfaces were measured using short probes to excite and receive surface waves. A layer of absorbing material was also placed above the samples to suppress space wave coupling. The measured transmission coefficients are given in Figure 4. We observe that the measured bandgap is slightly lower than that calculated using the equivalent circuit model. We also noted that bandgap of the REBG surface with $R=620 \Omega$ was deeper than that of $R=360 \Omega$. Of course, as expected, the REBG had a wider bandwidth than the unloaded EBG structure. In our design, we chose the REBG surface with $R=370 \Omega$ as it is capable of providing a good bandgap from 4.9 to $6 \mathrm{GHz}$ (with at least 10 $\mathrm{dB}$ surface wave attenuation).

\subsection{Absorption Coefficient}

It can be seen from Figure 2 that the REBG's surface impedance (at resonance) matches the free space impedance when $R=377 \Omega$. Thus, the plane wave reflection is very small for that value as verified in Figure 5. From Figure 5, we noted that the case of $R=370 \Omega$ had the strongest absorption at the resonance frequency of 5.67 $\mathrm{GHz}$ (absorption frequency).

Measurements for the REBG surface with $R=370 \Omega$ are also provided in Figure 5. These measurements were carried out using two horn antennas placed at a distance from the REBG sample and compared with the reflection from a metal sheet of the same size. A distance between the sample and antennas was fixed to $65 \mathrm{~cm}$ and the dis-

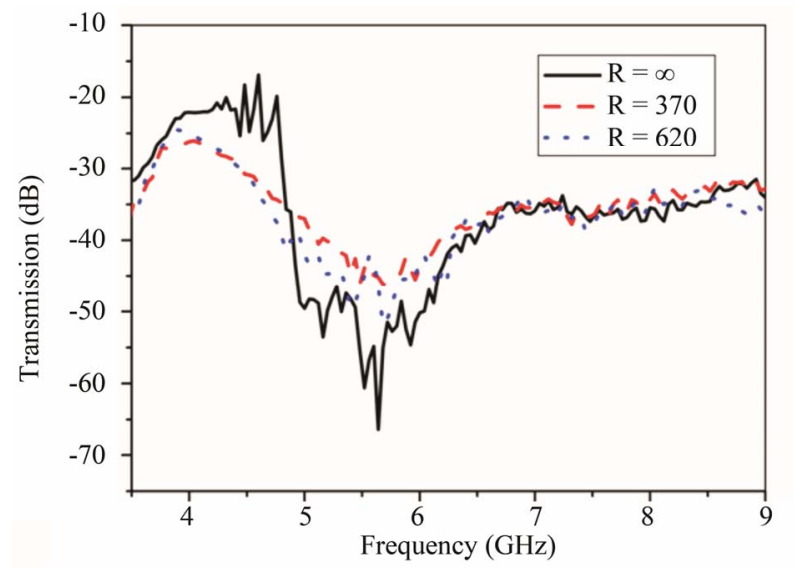

Figure 4. Measured transmission coefficient of the TM surface-wave across the REBG surface.

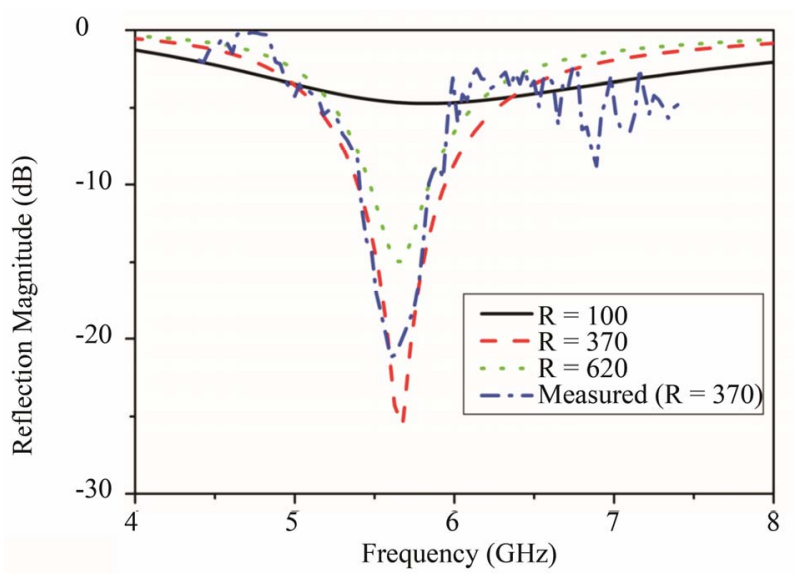

Figure 5. Numerical and experimental reflection coefficients of the REBG surface.

tance between these two antennas was $10 \mathrm{~cm}$. Absorbing material was placed between the antennas to reduce mutual coupling. The absorption property of the REBG surface is the dashed-dot line in Figure 5. We note that the reflection coefficient drops by $10 \mathrm{~dB}$ from $5.35-5.8$ $\mathrm{GHz}$, and reaches a minimum of $-26.4 \mathrm{~dB}$ at $5.64 \mathrm{GHz}$. Of importance is that the strongest absorption lies in the bandgap frequency.

\section{Slot Antenna Design Using REBG Structure}

\subsection{Tuning for Finite REBG Structures}

Diffraction at the finite REBG structure does have an impact on absorption and may detune the REBG resonance. This issue needs be considered when the REBG structure is adopted in antenna design.

To study this, a finite REBG structure with $N \times N$ elements ( $N$ varies from 3 to 16 ) was analyzed to obtain their absorption frequencies. As depicted in Figure 6, when the REBG aperture size decreases, the absorption frequency shifts toward a lower band. For the $3 \times 3$ structure, it is about $5.15 \mathrm{GHz}$, implying a $9.1 \%$ reduction as compared to the $5.67 \mathrm{GHz}$ of the infinite REBG surface. To achieve a desired absorption frequency, the parameters of the REBG structure (the substrate's dielectric constant and thickness, width of the patch and gap between them) must be optimized for a specific aperture size. Here the antenna was tuned to operate at the absorption frequency of the already designed finite REBG structure.

However, from Figure 6, we do note that the $10 \times 10$ array gives a resonance frequency close to that of an infinite array.

\subsection{Slot Antenna within the REBG Surface}

To examine the effectiveness of the REBG structure in 


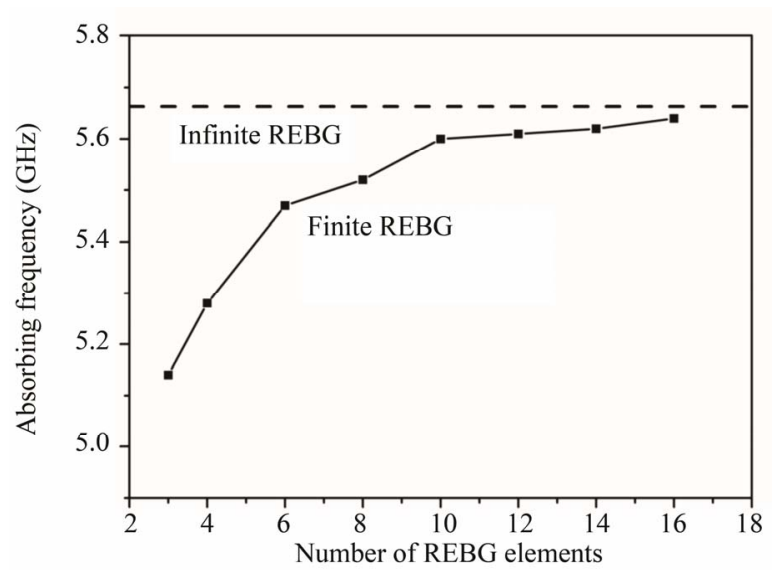

Figure 6. Absorption frequency of finite REBG structures for different elements in the array.

improving antenna performance, we considered a slot antenna [11] embedded within an REBG ground plane, as depicted in Figure 7. For this slot antenna design, REBG surface with $16 \times 16$ elements was chosen as we also considered the surface wave suppression properties. This finite REBG surface showed an absorption frequency of $5.65 \mathrm{GHz}$ (see Figure 6). The corresponding slot dimensions were $l=26 \mathrm{~mm}$ and $w=2 \mathrm{~mm}$. The slot was cut in the middle of a ground plane with the same size as the REBG surface. It was fed by a rectangular waveguide of $40.4 \mathrm{~mm} \times 20.2 \mathrm{~mm}$ in dimensions (see the photo in Figure 8).

Of importance is that the distance between the slot and REBG surface. As depicted in Figure 9, certain numbers of the center element were removed from the REBG surface, leaving a blank area of $L \times W$. This area is covered by metal to maintain the same boundary condition near the slot as in the metal ground plane. The width $W$ depends on how many columns of the REBG elements are removed. If $W$ is too small, strong coupling between the slot and REBG surface will dramatically influence the antenna impedance matching. Three cases with different W were considered. They are denoted as REBG1, REBG2 and REBG3, respectively, corresponding to the cases of 1, 2 and 3 columns of REBG elements were removed.

The antennas REBG1, REBG2 and REBG3 were simulated and their return loss is given in Figure 7. Also, the return loss of the antenna on a perfectly electrical conductor (PEC) ground plane is given for comparison. The slot antenna on the PEC ground plane resonated at $5.67 \mathrm{GHz}$ with a return loss of $-33 \mathrm{~dB}$.

The resonance frequencies of REBG1, REBG2 and REBG3 were $5.61 \mathrm{GHz}, 5.64 \mathrm{GHz}$ and $5.65 \mathrm{GHz}$, with a return loss of $-12.2 \mathrm{~dB},-16.6 \mathrm{~dB}$ and $-22.1 \mathrm{~dB}$, respectively. It should be noted that the resonance frequencies of REBG antennas did not change much and the return

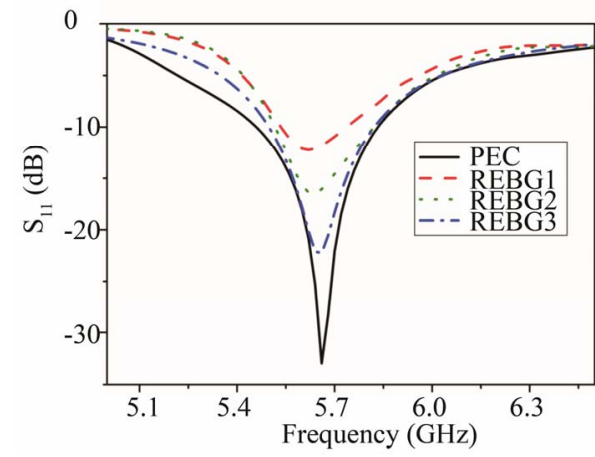

Figure 7. Return loss of slot antennas based on REBG surface with different number of center elements removed.

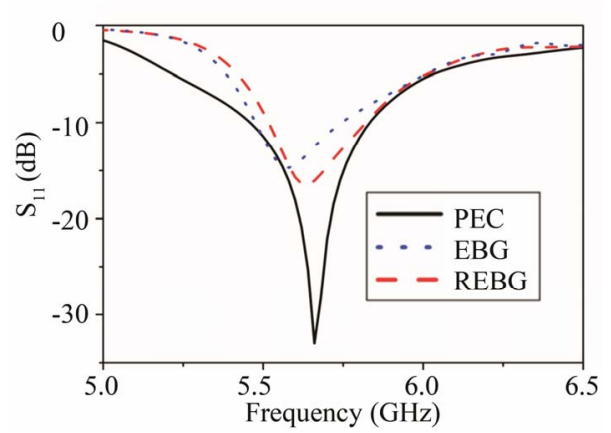

(a)

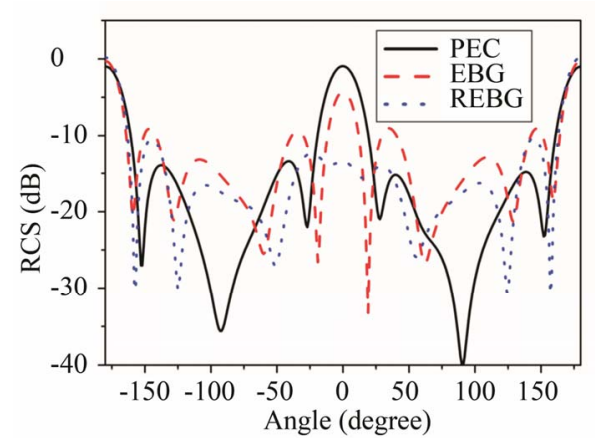

(b)

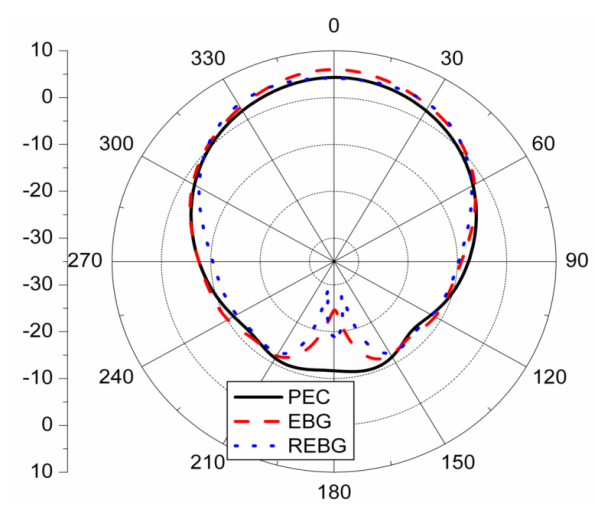

(c)

Figure 8. Performance of antennas on PEC, EBG and REBG ground planes: (a) return loss, (b) backward RCS, and (c) radiation pattern. 


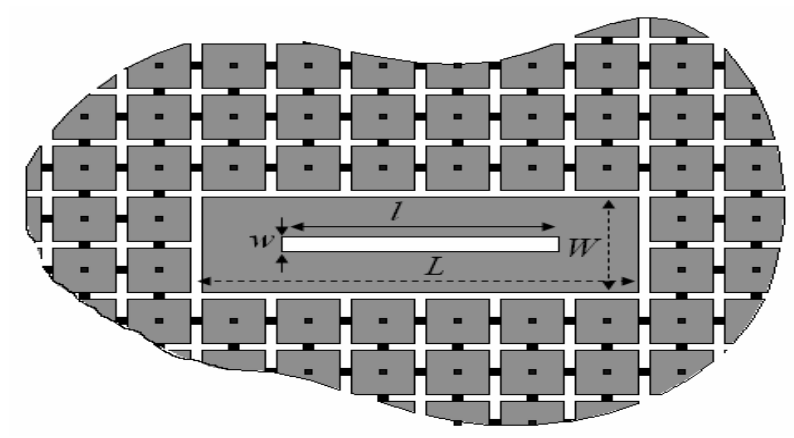

Figure 9. Illustration of a slot antenna in a REBG ground plane.

loss was good when 2 columns of REBG elements were removed.

The characteristics of gain, RCS and back lobes were also considered. We note that the REBG antenna's gain is lower than that of the antenna with PEC ground plane. This is due to the resistor loss. But as $W$ increases, the REBG antenna's gain increases. The back lobe levels of REBG2 and REBG3 have about $6-7 \mathrm{~dB}$ reduction as compared to the antenna on PEC ground plane (contrary for REBG1). It is also observed that the backscattered RCS of all of the REBG antennas is much lower than that of antenna on PEC ground plane. As shown in Table 1.

\subsection{Comparison of EBG and REBG Antennas}

To further evaluate the effectiveness of REBG structures in antenna design, we compared the performance of slot antennas on the REBG and EBG ground planes. It has been widely noted in literature that EBG structures can suppress surface waves in antennas and reduce side lobes.

The EBG version of REBG2 (by removing all resistors) was calculated as well and their performance is given in Figure 8. The return loss in Figure 8(a) shows that the EBG antenna has a worse matching and the frequency shift is larger. This is because the EBG surface partially reflects the surface waves back to the antenna so that the mutual coupling between the slot and REBG surface is stronger than that between the slot and REBG surface. As for the backscattered RCS in Figure 8(b), a $14 \mathrm{~dB}$ reduction (compared to PEC antenna) is observed for REBG antenna, but only $4 \mathrm{~dB}$ for EBG antenna. Clearly, the REBG structure can effectively reduce the antenna RCS. The antenna patterns are given in Figure 8(c). We note that the EBG antenna has a higher gain than the PEC and REBG antennas. The REBG antenna has a similar gain level to the PEC one. But, both of the REBG and EBG antennas has lower back lobes than the PEC one due to the surface wave suppression.

\subsection{Experimental Results}

Antenna REBG2 and a reference PEC antenna were fab-
Table 1. Simulated antenna performance (dB).

\begin{tabular}{ccccc}
\hline & REBG1 & REBG2 & REBG3 & PEC \\
\hline Gain & 3.0 & 4.1 & 4.4 & 4.8 \\
Back lobe & -8.1 & -18.8 & -17.8 & -11.6 \\
RCS & -22.3 & -16.2 & -16.1 & -1 \\
\hline
\end{tabular}
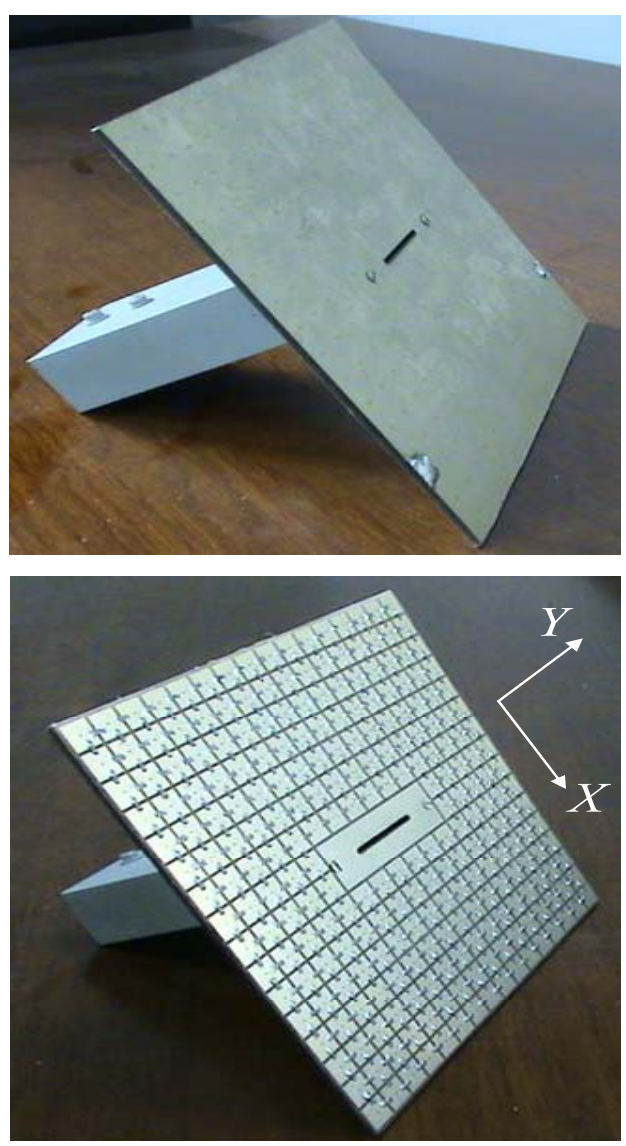

Figure 10. Photos of waveguide fed slot antennas with and without REBG ground plane.

ricated as shown in Figure 10. The radiation patterns and RCS of these two antennas were measured at $5.6 \mathrm{GHz}$ in the anechoic chamber. The measured gains were normalized to the PEC antenna gain (see Figure 11). It can be seen from Figure 11(a) that REBG antenna gain has a $0.3 \mathrm{~dB}$ drop than that of the PEC antenna. The back lobe is reduced by nearly $7 \mathrm{~dB}$.

Although there is a little gain drop for REBG antenna, its RCS is reduced dramatically. The measured RCS results were normalized to PEC antenna RCS as well (see Figure 11). In the normal direction, a reduction of 12.6 $\mathrm{dB}$ is obtained using the REBG ground plane. Moreover, more than $7 \mathrm{~dB}$ reduction is observed for angles in the range of $\pm 60^{\circ}$. The experimental photo is shown in Figure 12. 


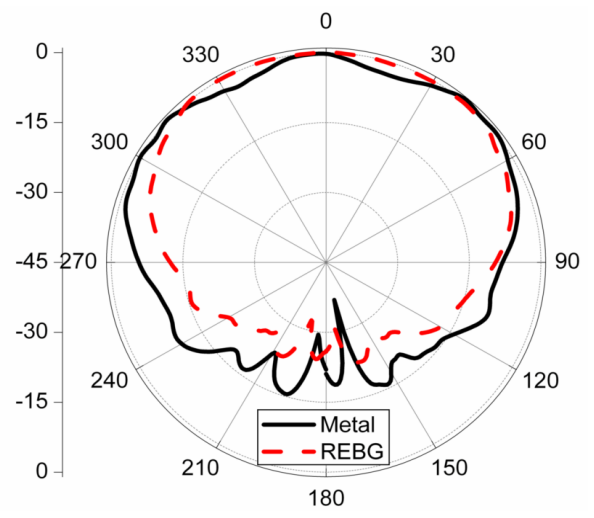

(a)

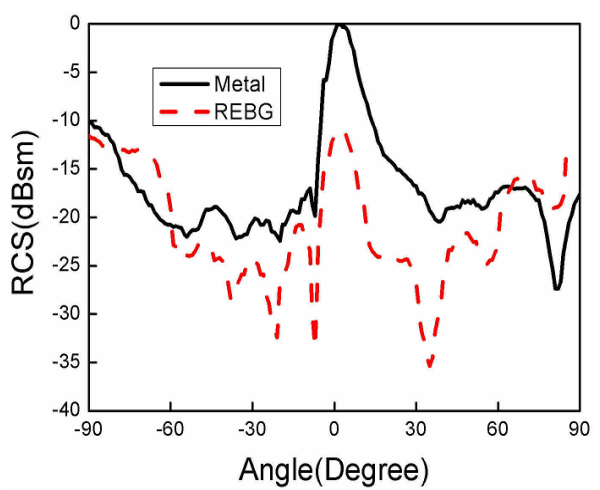

(b)

Figure 11. Measured radiation patterns of waveguide fed slot antennas on PEC and REBG ground planes: (a) radiation pattern, and (b) RCS.

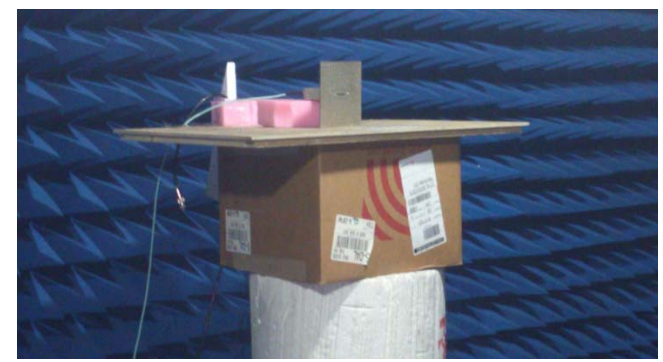

Figure 12. Photo of the measurement setup in the anechoic chamber.

\section{Conclusion}

Resistor loaded mushroom like EBG surface has been applied in slot antenna design. The antenna RCS can be significantly reduced and the antenna side lobe level be reduced as well. The REBG antenna gain is at the level of that on a PEC ground plane. Considering both of the radiation patterns and RCS, the total antenna performance is enhanced.

\section{REFERENCES}

[1] D. Sievenpiper, L. J. Zhang, R. F. J. Broas, N. G. Alexó- polous and E. Yablonovitch, "High-Impedance Electromagnetic Surfaces with a Forbidden Frequency Band," IEEE Transactions on Microwave Theory and Techniques, Vol. 47, No. 11, 1999, pp. 2059-2074. doi:10.1109/22.798001

[2] R. F. J. Broas, D. F. Sievenpiper and E. Yablonovitch, “A High-Impedance Ground Plane Applied to a Cellphone Handset Geometry," IEEE Transactions on Microwave Theory and Techniques, Vol. 49, No. 7, 2001, pp. 12611265.

[3] P. Maagt, R. Gonzalo, Y. C. Vardaxoglou and J. M. Baracco, "Electromagnetic Bandgap Antennas and Components for Microwave and (Sub)Millimeter Wave Applications," IEEE Transactions on Antennas and Propagation, Vol. 51, No. 10, 2003, pp. 2667-2677. doi:10.1109/TAP.2003.817566

[4] F. Yang and Y. Rahmat-Samii, "Micro-Strip Antennas Integrated with Electromagnetic Band-Gap Structures: A Low Mutual Coupling Design for Array Applications," IEEE Transactions on Antennas and Propagation, Vol. 51, No. 10, 2003, pp. 2936-2946. doi:10.1109/TAP.2003.817983

[5] I. Zeev, S. Reuven and B. Reuven, "Micro-Strip Antenna Phased Array with Electromagnetic Band-Gap Substrate," IEEE Transactions on Antennas and Propagation, Vol. 52, No. 6, 2004, pp. 1446-1453. doi:10.1109/TAP.2004.830252

[6] Y. Q. Fu and N. C. Yuan, "Elimination of Scan Blindness of Microstriop Phased Array Using Electromagnetic Bandgap Structures," IEEE Antennas and Wireless Propagation Letters, Vol. 3, No. 1, 2004, pp. 63-65. doi:10.1109/LAWP.2004.827891

[7] S. Collardey, A.-C. Tarot, P. Pouliguen and K. Mahdjoubi1, "Use of Electromagnetic Band-Gap Materials for RCS Reduction," Microwave and Optical Technology Letters, Vol. 44, No. 6, 2005, pp. 546-550. doi:10.1002/mop.20693

[8] D. J. Kern and D. H. Werner, "A Genetic Algorithm Approach to the Design of Ultra-Thin Electromagnetic Bandgap Absorbers," Microwave and Optical Technology Letters, Vol. 38, No. 1, 2003, pp. 61-64. doi:10.1002/mop.10971

[9] Q. Gao, Y. Yin, D. B. Yan and N. C. Yuan, "Application of Metamaterials to Ultra-Thin Radar Absorbing Material Design," Electronics Letters, Vol. 41, No. 17, 2005, pp. 936-937. doi:10.1049/el:20051239

[10] V. F. Fusco and S. W. Simms, "Textured Surface Slot Antenna with Reduced Radar Cross-Section," Electronics Letters, Vol. 43, No. 8, 2007, pp. 438-440. doi:10.1049/el:20070111

[11] Y. Li, H. Zhang, Y. Fu and N. Yuan, "RCS Reduction of Ridged Waveguide Slot Antenna Array Using EBG Radar Absorbing Material," IEEE Antennas and Wireless Propagation Letters, Vol. 7, 2008, pp. 43-45.

[12] M. Rahman and M. A. Stuchly, "Transmission Line-Periodic Representation of Planar Microwave Photonic Bandgap Structures," IEEE Antennas and Wireless Propagation Letters, Vol. 30, No. 1, 2001, pp. 15-19.

doi:10.1002/mop.1207 